\title{
Suplementación de dietas de gallinas de postura comercial con aceites de pescado de diferentes grados de refinación. Efectos productivos en las aves y en la calidad organoléptica de los huevos
}

\author{
Supplementation of commercial layer diets with different refined fish oils. \\ Effects on layer performance and sensory egg quality \\ S Cornejo ${ }^{a}$, H Hidalgo ${ }^{b}$, J Araya $^{a}, J_{\text {Pokniak }}{ }^{a}$

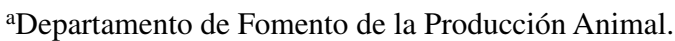 \\ bDepartamento de Patología Animal, Facultad de Ciencias Veterinarias y Pecuarias, Universidad de Chile.
}

\begin{abstract}
SUMMARY
The performance of 216 Leghorn layers and the sensory evaluation of their eggs were studied as a result of using different marine lipid sources (fish meal, oleine, crude fish oil and a refined fish oil, Alkitol-R-15®), in their diets. The birds were distributed randomly in 4 treatments: $\mathrm{T} 1=$ control, soy bean meal based diet; $\mathrm{T} 2=4 \%$ fish meal plus $3 \%$ oleine; $\mathrm{T} 3=4 \%$ fish meal plus $6 \%$ crude fish oil and T $4=4 \%$ fish meal plus $6 \%$ of refined fish oil concentrate ALKITOL-R-15®. Food and water were supplied ad libitum. The evaluated variables controlled were: laying percentage (LP), food consumption (FC), live weight (LW), egg weight (EW), egg mass (EM), feed conversion efficiency (FCE), mortality (M), gross margin, feeding cost per egg produced, egg flavour and aromatic quality, egg acceptability and preference. Diets with marine oils had a higher $(\mathrm{P}<0.05) \mathrm{EPA}+\mathrm{DHA}$ concentration than the T1 control. Main results showed that LP, LW, EW, EM, FCE and FC were similar between treatments $(\mathrm{P}>0.05)$ that included marine oils irrespective of the level in which they were included. Also, all these treatments had higher performances $(\mathrm{P}<0.05)$ than the control MS. The sensory evaluation indexes did not show any statistical differences $(\mathrm{P}>0.05)$ between the treatments tested. The inclusion of lipids from marine resources did not affect the layer performance nor the sensory evaluation of eggs and it should produce economic benefits to the producer.
\end{abstract}

Palabras clave: gallinas, aceite, rendimiento, evaluación sensorial.

Key words: hens, oil, performance, sensory evaluation.

\section{INTRODUCCION}

En las últimas décadas, a medida que han aumentado los indicadores socioeconómicos del país, se ha incrementado el interés de los consumidores por contar con productos alimenticios de origen animal de la mayor calidad y sobre todo, en el posible impacto de estos alimentos sobre la salud (Van Elswik y col 1998, García y col 2002, De Jong y col 2003), con lo que surge con fuerza el concepto de "alimentos nutracéuticos". Estos alimentos son productos que contienen nutrientes que por lo general son de bajo consumo, pero con reconocidos beneficios para la salud de la población. Un ejemplo son los denominados ácidos grasos poliinsaturados omega-3 o n-3 (AGPI n-3) de gran relevancia en el tratamiento y prevención de enfermedades cardiovasculares, hipertensión, diabetes, algunas formas de cáncer, y que, además, participan en el desarrollo del tejido nervioso encefálico y retinal (Eaton y col 1998, Zhang y Ross 2002). Las fuentes más ricas en AGPI n-3 son los peces de carnes oscuras. Sin embargo, a pesar de que nuestro país presenta una gran abundancia de este

Aceptado: 28.06.2007.

* Casilla 2, Correo 15, La Granja, Santiago, Chile; scornejo@uchile.cl recurso natural, la población sólo consume un promedio de $5 \mathrm{~kg} /$ habitante al año (Valenzuela 2005). Esto contrasta fuertemente con lo que acontece en países europeos y asiáticos (FAO 2000, Nakamura y col 2003).

Así, en nuestro medio, ha surgido la estrategia de incorporar AGPI n-3 a la dieta humana, empleando un "vehículo" como el huevo. Esto se logra alimentando a las aves de postura con insumos tales como harina de pescado (HP) y aceites de pescado (ACEIPES), que son capaces de replicar en el huevo su perfil de AGPI. La elección del huevo como vehículo se basa en sus excelentes características nutricionales y su precio, que lo convierten en uno de los productos más accesibles para todo tipo de estrato socioeconómico de la población. Además, los huevos no sólo se consumen en forma directa, sino que forman parte de un sinnúmero de preparaciones culinarias del diario vivir, lo que coayuda a masificar significativamente el respaldo nutricional deseado.

Sin embargo, el consumo nacional de huevos alcanza a 166 huevos/habitante/año (Asohuevo 2006) ${ }^{1}$, que representa un consumo de poco más de 3 huevos a la semana. Esta cifra muestra un aumento en el consumo de

ASOHUEVO. Asociación de Productores de Huevos. Boletín (en línea). www.asohuevo.cl (consulta 25/01/06) 
este producto desde 1980, pero la tendencia mundial es a una disminución, lo que en gran parte se debe a la alerta mundial generada por las autoridades de salud asociada al consumo de colesterol, presente en el huevo. Por otra parte, ya existen estudios donde se demuestra claramente que el aumento del consumo de huevos enriquecidos con AGPI n-3 no implica un aumento del colesterol sanguíneo de individuos sanos, aún más, en muchos casos, lo disminuye (Simopoulos 2000, McNamara 2002, Nakamura y col 2003, Norman y col 2004).

$\mathrm{Al}$ aumentar el aporte nutritivo de los huevos enriqueciéndolos con nutrientes como los AGPI n-3, vitaminas, antioxidantes y otros (Galobart y col 1999, Meluzzi y col 1999, Surai y Sparks 2000, Cubillos 2003), se le da un valor agregado al huevo que beneficiará a los consumidores y avicultores, quienes podrán lograr un valor agregado a su producción.

Estas estrategias alimentario-nutricionales pueden modificar la productividad de las aves (García y col 2002), por lo tanto, el objetivo del presente trabajo fue evaluar el efecto de la incorporación de diferentes fuentes de AGPI $\mathrm{n}-3$ en las dietas de gallinas ponedoras sobre su respuesta productiva y la calidad organoléptica de los huevos.

\section{MATERIAL Y METODOS}

Se emplearon 216 gallinas Leghorn Shaver, de segundo ciclo de postura, que fueron alojadas en el galpón experimental de la Facultad de Ciencias Veterinarias y Pecuarias de la Universidad de Chile. Las aves se asignaron aleatoriamente a cuatro tratamientos, de acuerdo al tipo y nivel de aceite de pescado incorporado a las dietas. Así, las aves fueron alimentadas con dietas isoenergéticas e isoproteicas: 1) Dieta Maíz-Soya sin insumos marinos (MS: T1); 2) Dieta control comercial con 4\% HP más $3 \%$ oleina (O) (HPO: T2); 3) dieta experimental con 4\% $\mathrm{HP}+6 \%$ aceite crudo de pescado (ACP) (HPACP: T3); 4) dieta experimental con $4 \% \mathrm{HP}+6 \%$ Alkitol R-15 ${ }^{\circledR 2}$, concentrado comercial blanqueado y estabilizado en su composición lipídica, con un aporte garantizado de 25-30\% de Eicosapentaenoico, (EPA) + Docosahexaenoico (DHA), (ALK) (HPALK: T4). Todas las dietas se presentan en el cuadro 1. Los insumos HP, O, ACP y ALK y las dietas empleadas fueron analizados químicamente para índice de peróxidos (IP), lípidos totales y perfil de ácidos grasos, siguiendo protocolos estandarizados (AOAC 1995).

El período experimental fue de ocho semanas durante las cuales se midieron los siguientes indicadores productivos: porcentaje de postura (PP), consumo de alimento (CA), eficiencia de conversión alimenticia (ECA), peso del huevo $(\mathrm{PH})$, peso vivo $(\mathrm{PV})$, masa de huevo $(\mathrm{MH})$ y mortalidad (M), considerando los valores promedios semanales de cada uno. Durante la quinta semana del ensayo las aves

\footnotetext{
2 ALKITOL. Química Industrial Spes. S.A.
}

Cuadro 1. Composición y aporte nutritivo de las dietas empleadas en el ensayo.

Composition and nutritive content of the diets throughout the assay.

\begin{tabular}{|c|c|c|c|c|}
\hline \multirow[b]{2}{*}{ Ingrediente (\%) } & \multicolumn{4}{|c|}{ Tratamientos } \\
\hline & MS: T1 & HPO: T2 & $\begin{array}{c}\text { HPACP: } \\
\text { T3 }\end{array}$ & $\begin{array}{c}\text { HPALK: } \\
\text { T4 }\end{array}$ \\
\hline Maíz, grano & 62,110 & 50,608 & 37,733 & 37,733 \\
\hline Sorgo, grano & 5,000 & 5,000 & 5,000 & 5,000 \\
\hline Soya, afrecho & 10,961 & 5,306 & 3,850 & 3,850 \\
\hline Maravilla, afrecho (pellet) & 0,000 & 5,000 & 5,000 & 5,000 \\
\hline Maravilla, afrecho (harina) & 5,000 & 5,000 & 5,000 & 5,000 \\
\hline Maíz, gluten & 4,030 & 1,000 & 1,000 & 1,000 \\
\hline Pescado, harina & 0,000 & 4,000 & 4,000 & 4,000 \\
\hline Oleina & 0,000 & 3,000 & 0,000 & 0,000 \\
\hline Pescado, aceite crudo & 0,000 & 0,000 & 6,000 & 0,000 \\
\hline Alkitol R-15® & 0,000 & 0,000 & 0,000 & 6,000 \\
\hline Trigo, afrechillo & 0,000 & 9,596 & 21,078 & 21,078 \\
\hline Conchuela & 8,842 & 9,289 & 9,420 & 9,420 \\
\hline Fosfato tricálcico & 3,322 & 1,690 & 1,440 & 1,440 \\
\hline $\mathrm{Sal}(\mathrm{NaCl})$ & 0,300 & 0,168 & 0,126 & 0,126 \\
\hline Antioxidante (ENDOX) & 0,013 & 0,013 & 0,013 & 0,013 \\
\hline Lisina & 0,160 & 0,070 & 0,077 & 0,077 \\
\hline Metionina & 0,162 & 0,160 & 0,163 & 0,163 \\
\hline Vitaminas pre-mix * & 0,050 & 0,050 & 0,050 & 0,050 \\
\hline Minerales pre-mix ${ }^{* *}$ & 0,050 & 0,050 & 0,050 & 0,050 \\
\hline TOTAL & 100,000 & 100,000 & 100,000 & 100,000 \\
\hline \multirow[t]{2}{*}{ Costo $(\$ / \mathrm{kg})^{* * *}$} & 81,830 & 75,850 & 77,300 & 89,960 \\
\hline & \multicolumn{4}{|c|}{ Aporte Nutritivo calculado } \\
\hline Proteína cruda $\%$ & 15,50 & 15,50 & 15,50 & 15,50 \\
\hline E. Metabolizable (kcal/kg) & 2.720 & 2.720 & 2.720 & 2.720 \\
\hline Calcio \% & 4,20 & 4,20 & 4,20 & 4,20 \\
\hline Fósforo Disponible \% & 0,38 & 0,38 & 0,38 & 0,38 \\
\hline Metionina \% & 0,43 & 0,44 & 0,44 & 0,44 \\
\hline Lisina \% & 0,75 & 0,75 & 0,75 & 0,75 \\
\hline Met + Cist. \% & 0,68 & 0,68 & 0,68 & 0,68 \\
\hline $\mathrm{Sal}(\mathrm{NaCl}) \%$ & 0,42 & 0,42 & 0,42 & 0,42 \\
\hline
\end{tabular}

* Premezcla Vitamínica: entrega por cada kg de dieta: Vit. A: 6000 UI; Vit. $\mathrm{D}_{3}$ : 1100 UI; Vit. E: 1,5 UI;

Vit. $\mathrm{K}_{3}: 1 \mathrm{mg}$; Vit. $\mathrm{B}_{2}: 2,5 \mathrm{mg}$; Vit. $\mathrm{B}_{12}: 0,003 \mathrm{mg}$; Niacina: $8 \mathrm{mg}$; Pantotenato de Calcio: $4 \mathrm{mg}$; Cloruro de Colina 50\%: $50 \mathrm{mg}$; Antioxidante: 2,5 mg.

** Premezcla Mineral: entrega por cada $\mathrm{kg}$ de dieta: Manganeso: $50 \mathrm{~g}$; Cobre: 2,5 mg; Yodo: 0,15 mg; Zinc: $15 \mathrm{mg}$; Hierro: $10 \mathrm{mg}$; Selenio: $0,05 \mathrm{mg}$.

*** Según costo de insumos alimenticios en diciembre 1999-enero 2000, informados por la granja avícola Arizona, empresa donde fueron elaboradas las dietas. ® Química Industrial SPES S.A.

fueron vacunadas contra Salmonella enteritidis (Laboratorio Biomune, USA), motivado por la realización de un estudio en paralelo a éste para evaluar la respuesta inmunológica de las gallinas. En forma complementaria, se estimaron las variables económicas: margen bruto $(\mathrm{MB})$ y costo 
alimentario (Cali) de los huevos producidos. El MB para cada tratamiento se calculó de acuerdo a la fórmula:

$\mathrm{MBi}=(\mathrm{Yi}-\mathrm{CAi})$

en donde:

$\mathrm{Yi}=$ ingreso $(\$)$

$\mathrm{CAi}=$ costo alimento $(\$ / \mathrm{kg}$ dieta $)$

$\mathrm{i}=$ Tratamientos $1-4$.

El ingreso se determinó considerando la producción semanal de huevos/tratamiento y luego para el total del periodo en estudio. Para ello, los huevos obtenidos en el muestreo semanal correspondiente se clasificaron comercialmente de acuerdo a su peso (g) en súper extra, extra, primera, segunda y tercera; así se ponderó cada una de las categorías comerciales por su correspondiente precio de mercado. El costo del alimento fue el señalado por el programa de formulación respectivo. El costo por concepto de gasto de alimento de los huevos producidos corresponde al producto de: ECA ( $\mathrm{kg}$ de alimento/ $\mathrm{kg}$ de huevos) por el precio del kilo de alimento.

Además, se realizó una evaluación sensorial al final del ensayo, utilizando huevos de los últimos 3 días de recolección del estudio, que fueron mantenidos en refrigeración, durante una semana, hasta el momento de la prueba. En ella, se incluyeron dos preparaciones culinarias: huevos duros y revueltos. Los últimos fueron cocinados en agua para evitar la influencia del aceite en los resultados. En la prueba participaron doce panelistas entrenados, que evaluaron el aroma, sabor y aceptabilidad de los huevos. Los evaluadores realizaron una prueba hedónica en base a una pauta no estructurada, que consiste en una línea de $15 \mathrm{~cm}$ de longitud que no presenta graduaciones en su extensión respecto de la característica a evaluar, pero sí entrega una valoración en el inicio (me disgusta mucho) y al final (me gusta mucho) (Campo 2005). Así, cada panelista debió marcar una línea vertical sobre la pauta, aproximándose a uno de los extremos de acuerdo a su propia percepción. Finalmente, para efectos de análisis, la pauta no estructurada presenta tres zonas imaginarias: a) rechazo $(1-7 \mathrm{~cm})$; b) indiferencia (de 7 a $8 \mathrm{~cm})$; c) aceptación $(8-15 \mathrm{~cm})$. Las variables productivas y sensoriales se describieron en base a sus promedios y desviaciones estándares, estudiándose el efecto de los tratamientos mediante un análisis de varianza (ANDEVA) usando un programa de SAS Institute (1989-1996). Las diferencias entre promedios específicos se establecieron empleando Tukey (Sokal y Rohlf 1981). Por último, se evaluó la preferencia por los huevos de cada tratamiento, para lo cual se entregó a cada panelista un set de cuatro muestras, provenientes de los cuatro tratamientos analizados; que fueron jerarquizados de mejor a peor, de acuerdo al grado de preferencia de cada uno. Así, se asignaron valores numéricos de 1 a 4 a este ordenamiento, donde 1 corresponde al mejor y 4 al peor, por lo que la sumatoria, suponiendo que una muestra obtuvo la primera preferencia $(=1)$ en todos los panelistas (12), entregará una valor final de 12, lo contrario que si fuese catalogada en último lugar
(4 x $12=48)$. Por lo tanto, un valor más cercano a 12 representa una mayor preferencia. Estos resultados fueron analizados según tablas para análisis de datos ranqueados de Newell y McFarlane (1987).

\section{RESULTADOS}

Los valores de composición química de los insumos y de las dietas empleadas se resumen en los cuadros 2a y $2 \mathrm{~b}$, respectivamente. Los valores de IP de los diferentes insumos son bajos, excepto el de ALK, que se eleva a 21,70 meq $\mathrm{O}_{2} / \mathrm{kg}$. Estos valores son el resultado de la progresiva mayor proporción de AGPI que poseen estos productos. Ello se refleja en el contenido de EPA + DHA de los insumos, que logran concentraciones de 14,82 22,11 - 28,33 y 29,94 (g/100 g de grasa), para HP, O, ACP y ALK, respectivamente, como también en las dietas, que mostraron porcentajes crecientes de extracto etéreo (base seca, \%) desde la dieta MS hasta la dieta HPALK. La misma tendencia se vio en los contenidos de EPA y DHA $(0,00-0,83-1,16-0,87$ para T1, T2, T3 у T4, respectivamente) donde el valor mostrado por $\mathrm{T} 4 \mathrm{que}$, sin explicación aparente, vio disminuida su concentración en estos ácidos grasos.

Las variables productivas se exponen en el cuadro 3. Los tratamientos que incluían lípidos de origen marino en las dietas mostraron valores superiores $(\mathrm{P}<0,05)$ al control, MS: T1. Cabe destacar que para todas las variables

Cuadro 2a. Composición química de los insumos de origen marino.

Chemical composition of sea origin ingredients.

\begin{tabular}{lcrrr}
\hline \multirow{2}{*}{ Indicadores } & \multirow{2}{*}{$\begin{array}{c}\text { Harina de } \\
\text { pescado }\end{array}$} & Oleína & \multicolumn{2}{c}{ Aceite } \\
& crudo de & ALKITOL \\
pescado & R-15 \\
\hline Humedad \% & 7,85 & 1,10 & 0,42 & 0,15 \\
Índice de peróxidos mEq $/ \mathrm{kg}$ & 3,60 & 1,50 & 7,50 & 21,70 \\
EPA g/100 de grasa & 9,06 & 11,93 & 16,77 & 19,06 \\
DHA g/100 de grasa & 5,76 & 10,18 & 11,56 & 10,88 \\
\hline
\end{tabular}

Cuadro 2b. Composición química de las dietas del estudio. Chemical composition of the diets used in the study.

\begin{tabular}{lcccc}
\hline \multirow{2}{*}{ Indicadores } & \multicolumn{4}{c}{ Dietas según tratamientos } \\
\cline { 2 - 5 } & MS: T1 & HPO: T2 & $\begin{array}{c}\text { HPACP: } \\
\text { T3 }\end{array}$ & $\begin{array}{c}\text { HPALK: } \\
\text { Humedad \% }\end{array}$ \\
Extracto etéreo \% base seca & 3,18 & 8,85 & 9,00 & 7,44 \\
EPA g/100 de grasa & 0,00 & 0,49 & 0,74 & 0,60 \\
DHA g/100 de grasa & 0,00 & 0,34 & 0,42 & 0,27 \\
EPA + DHA & 0,00 & 0,83 & 1,16 & 0,87 \\
Total AGPI* g/100 de grasa & 1,65 & 2,98 & 3,12 & 3,00 \\
\hline
\end{tabular}

* AGPI: ácidos grasos poliinsaturados. 
Cuadro 3. Indicadores productivos totales de las gallinas en el ensayo. Promedios \pm desviación estándar. Productive performance of the hens in the study. Averages \pm standard deviation.

\begin{tabular}{|c|c|c|c|c|}
\hline \multirow{2}{*}{ Indicadores } & \multicolumn{4}{|c|}{ Tratamientos } \\
\hline & MS: T1 & HPO: T2 & HPACP: T3 & HPALK: T4 \\
\hline Postura \% & $64,4 \pm 25,9^{b}$ & $75,9 \pm 14,9^{\mathrm{a}}$ & $73,9 \pm 15,7^{\mathrm{a}}$ & $75,2 \pm 15,5^{\mathrm{a}}$ \\
\hline Consumo Alimento $\mathrm{g}$ & $85,2 \pm 9,7^{b}$ & $99,2 \pm 6,7$ a & $99,5 \pm 4,4^{\text {a }}$ & $98,3 \pm 6,7^{\text {a }}$ \\
\hline Peso Vivo g & $1,54 \pm 0,14^{b}$ & $1,64 \pm 0,12^{\mathrm{a}}$ & $1,67 \pm 0,15^{\mathrm{a}}$ & $1,68 \pm 0,14^{\mathrm{a}}$ \\
\hline Peso Huevo g & $62,6 \pm 3,9^{b}$ & $65,8 \pm 4,6^{\mathrm{a}}$ & $64,7 \pm 5,1^{\text {a }}$ & $62,9 \pm 4,6^{b}$ \\
\hline Eficiencia Conversión Alimenticia & $2,70 \pm 1,7^{b}$ & $2,02 \pm 0,29^{a}$ & $2,11 \pm 0,32^{a}$ & $2,09 \pm 0,22 \mathrm{a}$ \\
\hline Masa Huevo & $40,30^{\mathrm{b}}$ & $50,10^{\mathrm{a}}$ & $47,90^{\mathrm{ab}}$ & $47,30^{\mathrm{ab}}$ \\
\hline Margen Bruto (\$) & 33.757 & 48.484 & 44.901 & 40.978 \\
\hline Costo alimentario $(\$)$ & 220,68 & 153,15 & 163,46 & 188,88 \\
\hline
\end{tabular}

a, b: promedios con diferentes letras en una misma fila indican diferencias significativas $(\mathrm{P}<0,05)$.

productivas no se produjeron diferencias entre los tres tratamientos con insumos de origen marino $(\mathrm{P}>0,05)$. La sola excepción fue el PH, donde el grupo HPALK fue estadísticamente semejante $(\mathrm{P}>0,05)$ al control MS y a su vez, inferior $(\mathrm{P}<0,05)$ a los tratamientos HPO y HPACP. El indicador MH, evaluado solamente como promedio general para todo el período experimental, mostró diferencias significativas $(\mathrm{P}<0,05)$, sólo entre el control (MS) y el grupo HPO.

Al observar los resultados económicos en el cuadro 3, fue el tratamiento 1 el que presentó el menor MB y mayor costo alimentario; en cambio, el tratamiento 2 mostró el mejor MB con el menor costo alimentario por kilo de huevos. En general, durante el ensayo, y a partir de la segunda semana, los tratamientos que incluían lípidos de origen marino fueron superiores en CA, PP, ECA y PV, al control. En el transcurso de la sexta semana del ensayo hubo una declinación de los indicadores, exceptuando el PV en los tratamientos HPO, HPACP y HPALK. Esta menor respuesta estuvo asociada a la vacunación contra Salmonella enteritidis, efectuada al final de la quinta semana. La mortalidad fue marginal alcanzando a cuatro aves $(1,85 \%)$ del total de las alojadas en el galpón.

Los resultados de la evaluación sensorial realizada (cuadro 4) pusieron en evidencia que ninguna de las preparaciones culinarias de los huevos de los tratamientos
Cuadro 5. Grado de preferencia de huevos duros y revueltos. Degrees of preference of boiled and scrambled eggs.

\begin{tabular}{lcccc}
\hline \multirow{2}{*}{ Huevos } & \multicolumn{4}{c}{ Tratamientos } \\
\cline { 2 - 5 } & MS: T1 & HPO: T2 & HPACP: T3 & HPALK: T4 \\
\hline Duros & $30^{1}$ & 28 & 27 & 35 \\
Revueltos & 25 & 32 & 31 & 32 \\
\hline
\end{tabular}

${ }^{1}$ Valores menores en una misma fila representan mayor preferencia.

utilizados fue rechazada para las tres variables analizadas. El análisis estadístico confirmó estos resultados al no evidenciar diferencias significativas entre los cuatro tratamientos $(\mathrm{P}>0,05)$. Más aún, casi todos los valores obtenidos se ubicaron en la zona de "aceptación" (puntajes entre 8 y $15 \mathrm{~cm})$. Algo similar ocurrió con la variable preferencia que no discriminó $(\mathrm{P}>0,05)$ a favor de algún tratamiento ni preparación en particular (cuadro 5), alcanzando todas las muestras valores de preferencia "intermedios", rango teórico entre 12 y 48.

\section{DISCUSION}

En Chile, la incorporación de insumos alimenticios de origen marino en las dietas de ponedoras no es nuevo, a

Cuadro 4. Aroma, sabor y aceptabilidad de huevos duros y revueltos. Promedios \pm desviación estándar.

Sensory score evaluation of boiled and scrambled eggs. Averages \pm standard deviation

\begin{tabular}{llrrrr}
\hline \multirow{2}{*}{ Huevos } & \multirow{2}{*}{ Variable } & \multicolumn{4}{c}{ Tratamientos } \\
\cline { 3 - 5 } & & MS: T1 & HPO: T2 & HPACP: T3 & HPALK: T4 \\
\hline \multirow{3}{*}{ Duros } & Aroma & $8,70 \pm 3,2$ & $7,37 \pm 3,2$ & $8,23 \pm 2,4$ & $7,53 \pm 3,3$ \\
& Sabor & $8,75 \pm 3,3$ & $9,65 \pm 2,4$ & $8,15 \pm 1,9$ & $9,33 \pm 3,5$ \\
& Aceptabilidad & $9,60 \pm 2,7$ & $10,30 \pm 3,5$ & $8,15 \pm 3,3$ & $8,32 \pm 3,5$ \\
\multirow{3}{*}{ Revueltonyyyy} & Aroma & $8,41 \pm 2,9$ & $8,54 \pm 2,9$ & $8,30 \pm 2,9$ & $7,81 \pm 2,7$ \\
& Sabor & $8,96 \pm 2,4$ & $8,24 \pm 2,8$ & $9,5 \pm 2,5$ & $8,7 \pm 2,7$ \\
& Aceptabilidad & $9,43 \pm 2,6$ & $9,8 \pm 1,8$ & $10,2 \pm 2,3$ & $9,13 \pm 2,7$ \\
\hline
\end{tabular}


pesar que en la actualidad, debido a condiciones coyunturales por el alto precio de las HP, este alimento casi no forma parte de las dietas avícolas comerciales, pero sí se emplean ACEIPES con diferentes grados de refinación y precios. Por consiguiente, era interesante evaluar altos niveles de incorporación de ACEIPES (6\%) versus niveles más bajos usados casi de rutina (3\%) y estudiar el comportamiento de un producto industrial con refinado especial y concentrado en EPA y DHA, también incorporado en un $6 \%$ de la dieta.

Los resultados de las evaluaciones químicas efectuadas a insumos y dietas fueron los esperados para alimentos con altos porcentajes de AGPI, entre ellos, la presencia de un alto nivel de peróxidos en ALK. Estos insumos lipídicos altamente insaturados tienen un gran riesgo oxidativo y deben ser "protegidos", con la incorporación de antioxidantes (ya sea uno o en combinaciones, naturales y/o de síntesis), en directa relación al nivel de inclusión de los insumos lipídicos insaturados a las dietas, tal como lo señalan Aymond y Van Elswyk (1995), Meluzzi y col (1999), Galobart y col (1999) y Surai y Sparks (2000). En el presente estudio se empleó un solo nivel de antioxidante en todas las dietas (cuadro 1) y sería aconsejable incluir el antioxidante de acuerdo al nivel de AGPI, lo que se debería considerar en futuras investigaciones.

Las respuestas productivas de las gallinas (cuadro 3 ) no fueron afectadas negativamente, al compararlas con el grupo control, por la inclusión de hasta un $6 \%$ de aceites marinos en sus dietas (HPACP y HPALK), y los resultados son coincidentes con lo informado por Hargis y col (1991), Van Elswyk y col (1992). Sin embargo, Suk y col (1994), Meluzzi y col (1999) publican PP y PH inferiores en gallinas a las cuales se les suministró un concentrado de EPA + DHA a niveles del 10\% de sus dietas. Cuando este suplemento se rebajó a un 5\%, el efecto negativo permaneció sólo en el PH. Por otra parte, Nash y col (1995) y Baucells y col (2000) señalan que al incluir insumos de origen marino en las dietas de gallinas de postura obtienen valores decrecientes en el $\mathrm{PH}$. El indicador $\mathrm{MH}$, se comportó de acuerdo a los valores de PP y PH, que se factorizan para su cálculo. Sólo el tratamiento HPO se mostró estadísticamente diferente y superior a los demás $(\mathrm{P}<0,05)$. Así, esta característica no fue afectada negativamente por la inclusión de ACEIPES en las dietas, lo que se contrapone a lo informado por González-Esquerra y Leeson (2000), quienes señalan que el incremento en la inclusión de aceite de arenque en dietas de ponedoras, determina una MH cada vez menor $(\mathrm{P}<0,05)$.

Todas las variables mostraron un descenso en la semana 6 del ensayo, cuya intensidad dependió de cada tratamiento, pero fue especialmente evidente en el grupo MS control, tanto para PP, CA y ECA. El consumo de alimento tuvo una gran recuperación, especialmente en los tratamientos que incluían productos de origen marino. Aunque se sabe que las aves tienen menos desarrollado el sentido del gusto, es posible que el comportamiento del CA estuviese asociado a un efecto positivo sobre la aceptabilidad ejercida por los aceites marinos presentes en las dietas que los incluían. Esto coincide con resultados informados por Baucells y col (2000) al emplear un máximo de $4 \%$ de aceite de pescado en dietas de ponedoras. Este cambio en el comportamiento productivo de las ponedoras estuvo definitivamente asociado al manejo experimental de vacunación contra Salmonella enteritidis, que se efectuó durante el ensayo.

El menor impacto de la vacunación observado en los tratamientos experimentales, con una mejor suplementación en AGPI, en particular en EPA y DHA, pudiera asociarse a la presencia de estos ácidos grasos que habrían contribuido a compensar el estrés vacunal, haciendo menos agresivo el estrés inmunológico y por lo tanto, menos perjudicial sobre los rendimientos productivos de las aves. Así lo sugieren Korver y Klasing (1995) y Klasing y Korver (1998), al señalar que los AGPI n-3 (EPA y DHA) modulan la respuesta inmune tanto celular como humoral, al modificar la producción de interleuquina-1 y disminuir la de prostaglandina E-2, lo que reduce la intensidad de la respuesta inflamatoria vacunal, beneficiando la respuesta productiva.

Los panelistas profesionales, que realizaron la evaluación sensorial de los huevos, no evidenciaron la existencia de aromas y sabores "a pescado", ligados a la presencia de aceites de origen marino, que pudieran haber sido traspasados al huevo. Así, la aceptabilidad de estos huevos fue satisfactoria tanto para la preparación culinaria "huevos duros" como para "huevos revueltos". Esta evaluación coincide con lo reportado por Maurice (1994), Marshall y col (1994), quienes no logran encontrar rechazos organolépticos a huevos de gallinas alimentadas con insumos de origen marino. Por otra parte, van Elswyk y col (1992), Oh y col (1994), González-Esquerra y Leeson (2000) informan la presencia de sabores "a pescado" en los huevos evaluados cuando se emplearon entre 2 y $6 \%$ de ACEIPES. Las pruebas organolépticas efectuadas en el presente estudio permiten señalar que la inclusión de hasta un $6 \%$ de los insumos de origen marino, aún de aquéllos menos refinados que el ALK ${ }^{\circledR}$, no afecta negativamente ni la aceptabilidad ni señala claras preferencias por ninguno de los huevos provenientes de los tratamientos probados.

La información reunida en este trabajo puso de manifiesto que la incorporación de aceites marinos no afectó negativamente la respuesta productiva de las aves como tampoco modificó la aceptabilidad del producto y subsidiariamente entregó una protección al estrés posvacunal de las gallinas. Además, de acuerdo a la evaluación económica efectuada, también tendría ventajas comparativas la inclusión de estos recursos a la dieta de las ponedoras.

Finalmente, se debe enfatizar que el consumidor de estos productos no presentaría objeción alguna al alimentarse con ellos, por lo que son las empresas avícolas de postura tanto como las plantas elaboradoras de ACEIPES para uso avícola, las que deben incentivar su empleo, con especial énfasis a la condición de ofrecer huevos como un "alimento nutracéutico", con un alto valor nutricional 
agregado para el hombre; como ocurre en Brasil (Shinoda 2000), donde se están produciendo y comercializando huevos enriquecidos nutricionalmente. En el país, se ha comenzado a ofrecer estos huevos enriquecidos con AGPI n-3 y /o con vitamina E.

\section{RESUMEN}

Se evaluó el efecto sobre: porcentaje de postura (PP), consumo (CA), peso vivo (PV), peso del huevo $(\mathrm{PH})$, masa de huevo $(\mathrm{MH})$, conversión alimentaria (ECA), mortalidad (M), margen bruto (MB) y costo alimentario (Cali) del empleo de: harina de pescado: HP; oleina: O; aceite crudo de pescado: ACP y aceite de pescado refinado: ALKITOLR-15@: ALK, en dietas de gallinas ponedoras comerciales. Además, se efectuó una evaluación sensorial de los huevos incluyendo: aroma, sabor, aceptabilidad y preferencia. Se utilizaron 216 gallinas Leghorn Shaver, durante 8 semanas, distribuidas en cuatro tratamientos: T1 $=0 \%$ HP y aceites, $\mathrm{T} 2=4 \% \mathrm{HP}+3 \% \mathrm{O} ; \mathrm{T} 3=4 \% \mathrm{HP}+6 \% \mathrm{ACP} ; \mathrm{T} 4=4 \% \mathrm{HP}+$ $6 \%$ ALK. El índice de peróxidos (IP) fue de 1,5; 7,5 y 21,7 meq $\mathrm{O}_{2} / \mathrm{kg}$ para O, ACP y ALK, respectivamente. La HP "prime" presentó un IP de 3,6. Las dietas mostraron porcentajes de EPA + DHA de 0,00 - 0,83 - 1,16 y 0,87, desde T1 a T4, respectivamente. Los indicadores PP, CA, $\mathrm{PV}, \mathrm{PH}$, ECA y MH fueron semejantes entre T2, T3 y T4 $(\mathrm{P}>0,05)$ siendo superiores, en general $(\mathrm{P}<0,05)$ al $\mathrm{T} 1$. El T2 generó el mayor MB y el menor Cali. La evaluación sensorial de los huevos (duros y revueltos) no mostró diferencias significativas $(\mathrm{P}>0,05)$ entre los tratamientos para ambas preparaciones. Se concluye que la incorporación de lípidos marinos no afecta el rendimiento productivo de las aves ni provoca efectos sensoriales adversos en los huevos y ofrecería ventajas económicas al productor.

\section{AGRADECIMIENTOS}

Los autores agradecen a las empresas "Química Industrial SPES S.A." y "Granja Avícola Arizona", cuyos aportes permitieron la realización del presente estudio.

\section{REFERENCIAS}

AOAC, Association of Official Analytical Chemists. 1995. Methods of Analysis. Washington DC, USA.

Aymond W, M van Elswyk. 1995. Yolk thiobarbituric acid reactive substances and n-3 fatty acids in response to whole and ground flaxseed. Poult Sci 74, 1388-1394.

Baucells M, N Crespo, A Barroeta, S López-Ferrer, M Grashorn. 2000. Incorporation of different polyunsaturated fatty acids into eggs. Poult Sci 79, 51-59.

Campo M. 2005. Consumidores. En: Cañeque V, Sañudo C (eds). Estandarización de las metodologías para evaluar la calidad del producto (animal vivo, canal, carne y grasa) en los rumiantes. Monografías INIA: Serie Ganadera. N ${ }^{\circ}$ 3-2005. INIA, Madrid, España, Pp 409-422.

Cubillos M. 2003. Incorporación de ácidos grasos omega-3, selenio orgánico y vitamina $E$ en las dietas de gallinas de postura: 1.-Indicadores productivos y respuesta inmune. Memoria de titulación, Escuela de Ciencias Veterinarias, Universidad de Chile, Santiago, Chile.

De Jong N, M Ocké, H Branderhorst, R Friele. 2003. Demographic and lifestyle characteristics of functional food consumers and dietary supplement users. British J Nutr 89, 273-281.

Eaton S, S Eaton, A Sinclair, L Cordain, N Mann. 1998. Dietary intake of long chain polyunsaturated fatty acids during the paleolithic. World Rev Nutr Diet 83, 12-23.

FAO, Food and Agriculture Organization of the United Nations. 2000. Food Balance Sheets.
Galobart J, A Barroeta, M Baucells, F Guardiola. 1999. Vitamin E levels and lipid oxidation in n-3 fatty acids enriched eggs. Proceedings VIII European Symposium, Quality Eggs and Egg Products, Bologna, Italia, Pp 19-23.

García C, S Cornejo, C Albala. 2002. Enriched eggs for human consumption and the feeding pattern of layers. In: Watson R (ed). Eggs and Health Promotion. Iowa State Press, Ames, USA, Pp 155-170.

González-Esquerra R, S Leeson. 2000. Effect of feeding hens regular or deodorized arenque oil on production parameters, fatty acid profile and sensory quality of eggs. Poult Sci 79, 1597-1602.

Hargis P, M van Elswyk, B Hargis. 1991. Dietary modification of yolk lipid with arenque oil. Poult Sci 70, 874-883.

Klasing K, D Korver. 1998. The role of dietary long chain polyunsaturated fatty acids in modulating the immune response of broilers. En: Cornejo S, Hidalgo H (eds). VI Seminario Internacional de Patología y Producción Avícola. Santiago, Chile. Pp 187-199.

Korver D, K Klasing. 1995. Interactions between nutrition and the immune system with emphasis on fatty acids. XIV Congreso Latinoamericano de Avicultura, Santiago, Chile, Pp 22-29.

Marshall A, A Sams, M van Elswyk. 1994. Oxidative stability and sensory quality of stored eggs from hens fed $1.5 \%$ arenque oil. J Food Sci 59, 561-563.

Maurice D. 1994. Dietary fish oils: Feeding to produce designer eggs. Feed Manag 45, 29-32.

Mc Namara D. 2002. Eggs, plasma colesterol and heart Disease Risk. In: Watson R (ed). Eggs and Health Promotion. Iowa State University Press, Ames, Iowa, USA, Pp 71-81.

Meluzzi A, F Sirri, N Tallarico, L Vandi. 1999. Dietary vitamin E in producing eggs enriched with n-3 fatty acids. Proceedings VIII European Symposium, Quality Eggs and Egg Products, Bologna, Italia, Pp 153-159.

Nakamura T, A Azuma, T Kuribayashi, H Sugihara, S Okuda, M Nakagawa. 2003. Serum fatty acid levels, dietary style and coronary heart disease in three neighbouring areas in Japan: the Kumihama study. Br J Nutr 89, 267-272.

Nash D, R Hamilton, H Hulan. 1995. The effect of dietary herring meal on the omega-3 fatty acid content of plasma and egg yolk lipids of laying hens. Can J Anim Sci 75, 247-253.

Newel G, J MacFarlane. 1987. Expanded tables for multiple comparisons procedures in the analysis of ranked data. J Food Sci 52, 1721-1725.

Norman L, C Shaw, C Fink, A Awad. 2004. Combination of phytosterols and omega-3 fatty acids: a potential strategy to promote cardiovascular health. Curr Med Chem Cardiovascular and Hematological Agents 2, 1-12.

Oh S, C Lin, J Ryue, D Bell. 1994. Eggs enriched in n-3 fatty acids as a wholesome food. J Appl Nutr 46, 14-25.

Shinoda W. 2000. Mercadeo de huevos omega-3 en Brasil. Industria Avícola Abril, 6-10.

Simopoulos A. 2000. Symposium: Role of poultry products in enriching the human diet UIT N-3 PUFA. Human requirement for $\mathrm{n}-3$ polyunsaturated fatty acids. Poult Sci 79, 961-970.

Sokal R, F Rohlf. 1981. Biometry. $2^{\text {nd }}$ ed. Freeman and Co. (eds). New York, USA, $859 \mathrm{p}$.

Suk Y, H Chia-Hong, R Jehong, E Dean. 1994. Eggs enriched with n-3 fatty acids as a wholesome food. J Appl Nutr 46, 14-25.

Surai P, N Sparks. 2000. Designer egg production and evaluation. Conferencias y Resúmenes XI Congreso Nacional de Medicina Veterinaria, Santiago, Chile, Pp 45-50.

Valenzuela A. 2005. El salmón: un banquete de salud. Rev Chil Nutr 32, 8-17.

Van Elswyk M, A Sams , P Hargis. 1992. Composition, functionality and sensory evaluation of eggs from hens fed dietary menhaden oil. J Food Sci 57, 342-349.

Van Elswyk M, S Hatsh, G Stella, P Mayo, K Kubena. 1998. Poultry-based alternatives for enhancing the n-3 fatty acid content of american diets. World Rev Nutr Diet 83, 102-115.

Zhang J, R Ross. 2002. Health effects of docosahexaenoic acid (DHA)enriched eggs. In: Watson R (ed). Eggs and Health Promotion. Iowa State Press, Ames, USA, Pp 123-132. 